\title{
PERIOD VARIATION OF BW VUL
}

\author{
JIANG SHI-YANG \\ Beijing Astronomical Observatory, Chinese Academy of Sciences
}

Due to the large fluctuation of the time of light maxima, in each observational season only very few observations is very dangerous for period variation research. Especially when the observation is not accurate enough such as for the early radial velocity data. Of course parts of the fluctuation are real which are caused by the chaotic behavior of the complicated pulsation. Generally the pulsation is quite periodic but is not like a timer such as the oscillation of the quartz or the rotating of the pulsar, all of these as much simple.

The period increasing rate of $\mathrm{BW}$ Vul from our results is 0.34 micron days per year which makes a evolution time scale about 3 million years. For a star with mass of 10 solar mass, the life age on the main sequence is about 10 million years. So BW Vul should not be very far away from the main sequence.

Our formula for calculate the time of light maxima is

$$
\begin{aligned}
& T_{\max }=\text { HJD2428802.7250+0.201027291 +9.35 } \times 10^{-11} E^{2} \\
& \pm 16 \quad 56 \quad 5
\end{aligned}
$$

This formula to fit the parabolic curve in figure 1 is almost exactly the same for to fit that in figure 2. Because after 1967 all the observations are very good, the situation in figure 2 should be very reliable. So the figure 1 also should be very reliable.

In figure 3 there are 6 points rather far away from the parabolic curve. The first one was given by Huffer in 1937 with very low time resolution so the light curve shape is quite different with our recent observation. So the reliability is very bad. The 3 points given by Cester in 1957 and 2 points given by McNamara in 1960 are not only have rather larger errors but really is too few points in each season. So we can not use them as a evidence to show some kind of special period variation. 


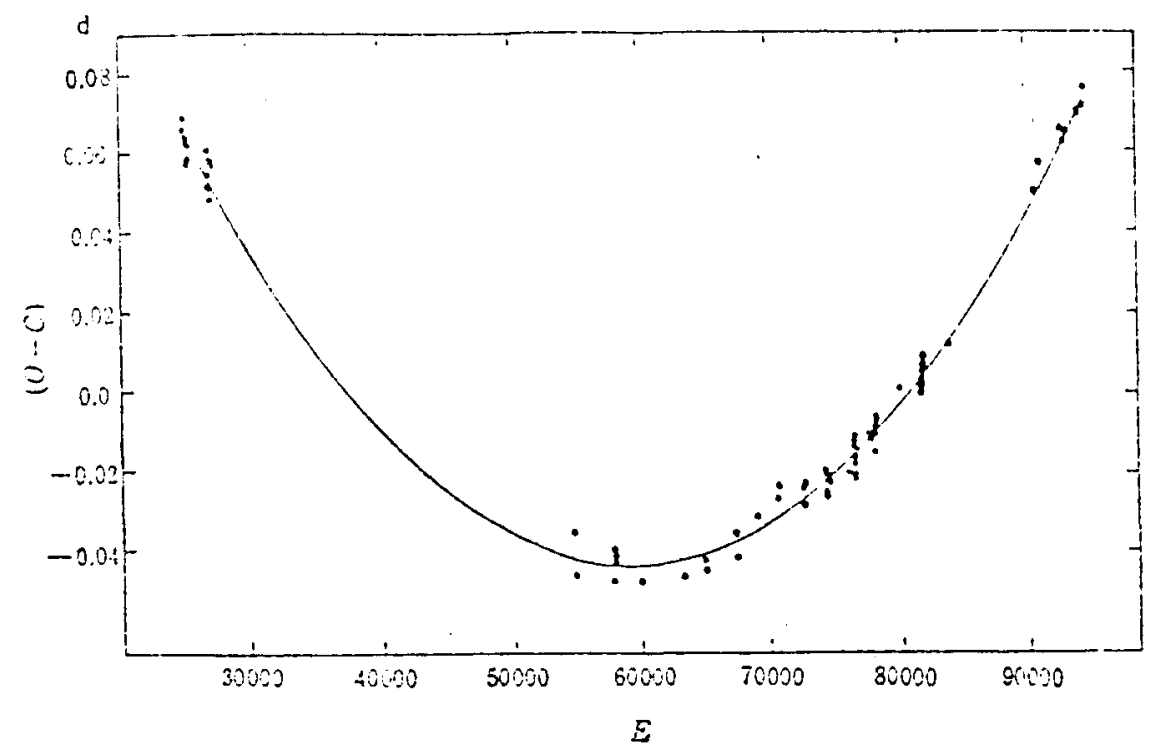

Fig. 1. The $(O-C)$ Curve for all the reliable photoelectric measures time of light maxima.

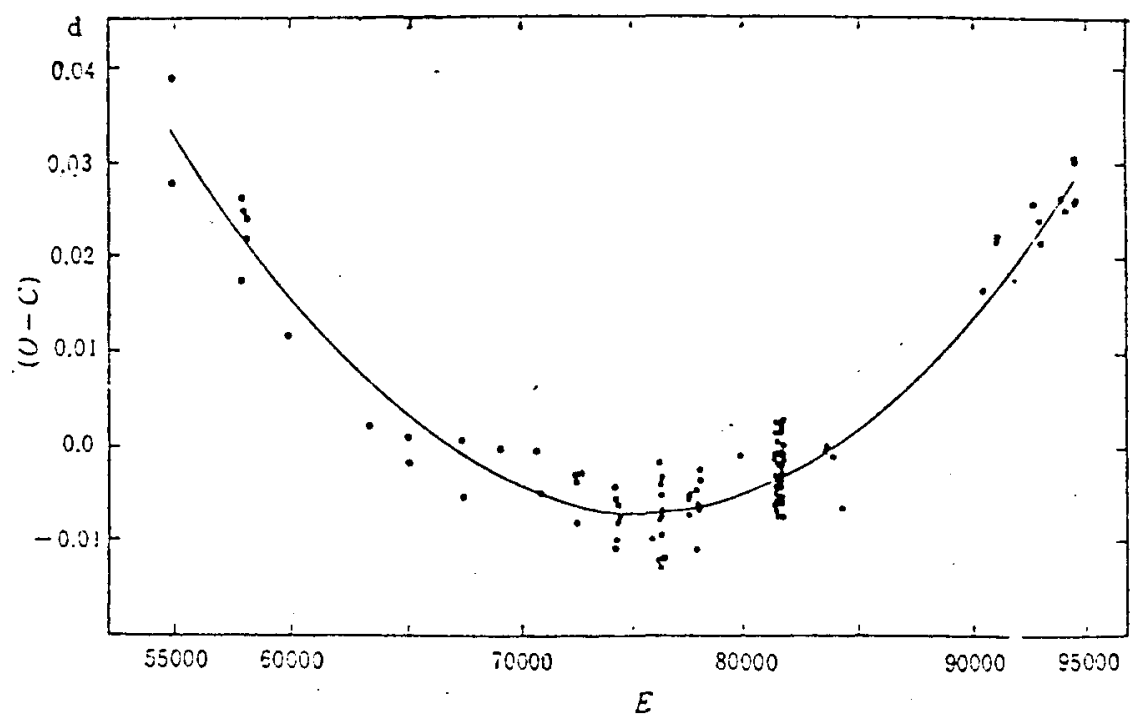

Fig. 2. The $(O-C)$ Curve for all the time of light maxima after 1967 . 


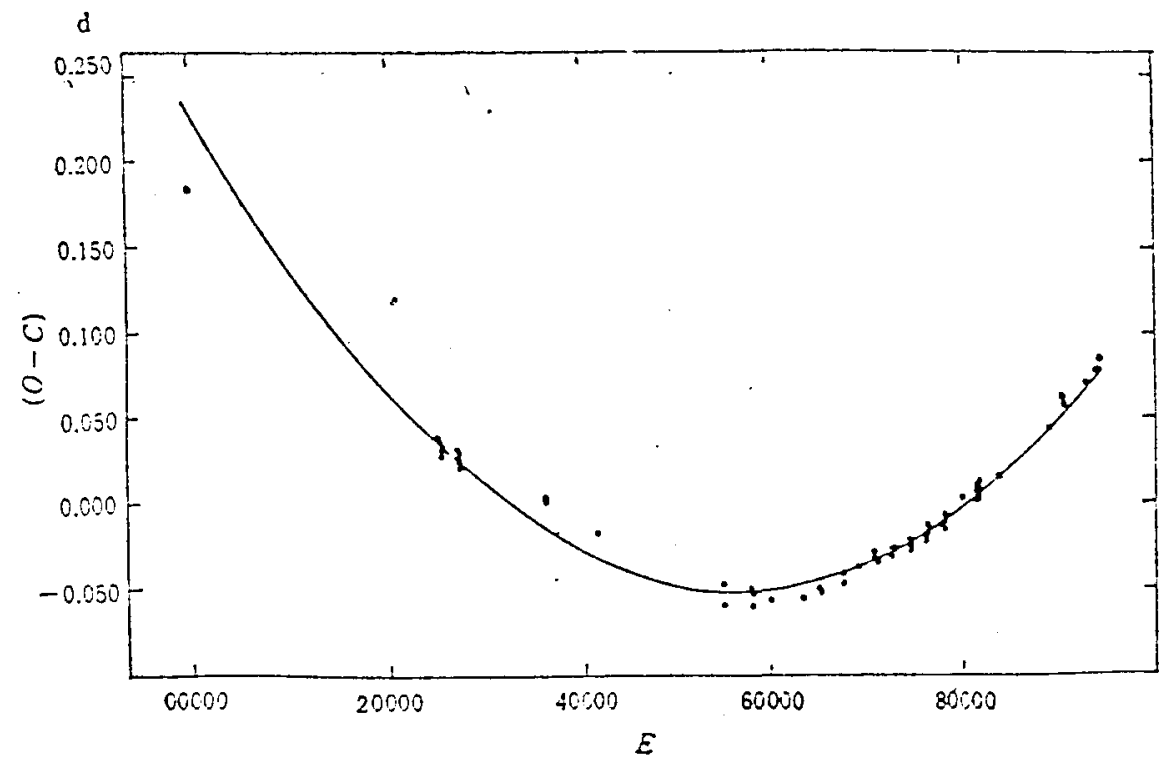

Fig. 3. The $(O-C)$ Curve for all the time of light maxima except that calculated from the radial velocity data. 